\title{
Structural and dipolar fluctuations in liquid water: A Car-Parrinello molecular dynamics study.
}

\author{
Ioannis Skarmoutsos ${ }^{\dagger, ~ * 1}$, Marco Masia ${ }^{\ddagger}, *$, and Elvira Guardia ${ }^{\S, *}$
}

\footnotetext{
${ }^{\dagger}$ Department of Earth Sciences, University College London, Gower Street, London WC1E 6BT, United Kingdom,e-mail: i.skarmoutsos@ucl.ac.uk

‡ Dipartimento di Chimica e Farmacia, Universita degli Studi di Sassari, Istituto Officina dei Materiali del CNR, UOS SLACS, Via Vienna 2,07100 Sassari, Italy, email: marco.masia@uniss.it

${ }^{\S}$ Departament de Física, Universitat Politecnica de Catalunya, Campus Nord B4-B5, Barcelona 08034, Spain, email: elvira.guardia@upc.edu
}

\begin{abstract}
A Car-Parrinello molecular dynamics simulation was performed to investigate the local tetrahedral order, molecular dipole fluctuations and their interrelation with hydrogen bonding in liquid water. Water molecules were classified in three types, exhibiting low, intermediate and high tetrahedral order. Transitions from low to high tetrahedrally ordered structures take place only through transitions to the intermediate state. The molecular dipole moments depend strongly on the tetrahedral order and hydrogen bonding. The average dipole moment of water molecules with a strong tetrahedral order around them comes in excellent agreement with previous estimations of the dipole moment of ice Ih molecules.
\end{abstract}

\section{Graphical Abstract}

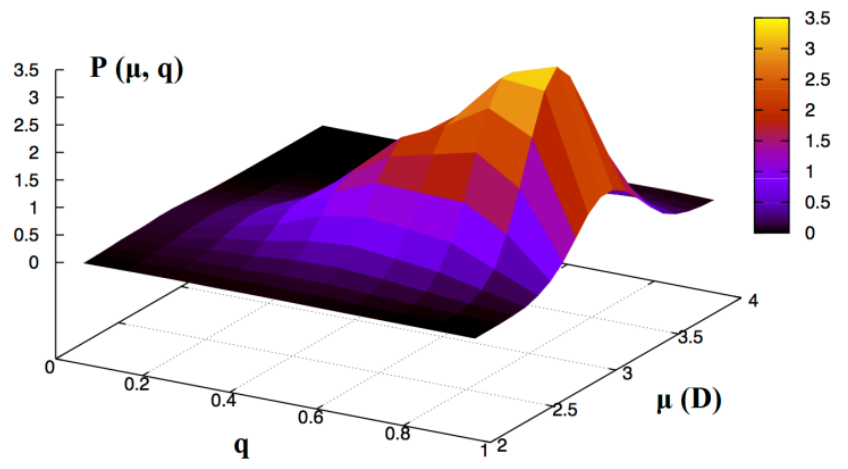

\footnotetext{
${ }^{1}$ Present address: Department of Physics and Astronomy, University College London, Gower Street, London WC1E 6BT, United Kingdom
} 
Keywords: Liquid water, Car-Parrinello, molecular dynamics, tetrahedral order, dipole moment, hydrogen bonding

\section{Highlights}

- The local tetrahedral order and dipolar fluctuations in liquid water were studied.

- Car-Parrinello quantum molecular dynamics simulations have been performed.

- The dipole moments depend strongly on the tetrahedral order and hydrogen bonding.

- High tetrahedrally-ordered water and ice Ih molecules have similar dipole moments. 


\section{Introduction}

The properties of liquid water have attracted the attention of the scientific community due to its crucial role and importance in a wide range of chemical, biological and geological processes [1,2]. A detailed understanding of the properties of water at several thermodynamic conditions is therefore essential and at the same time demanding due to its complex, and often characterized as anomalous, behavior [3].

There have been a number of claims about the nature of water as a liquid that seem to contradict and challenge traditional views [4,5]. According to these claims, water is not the tetrahedral network that it is traditionally regarded as. The deviation of the average coordination of water from four (which is the number that the tetrahedral coordination of ice and the nature of the hydrogen bond suggest) has also been a subject of strong debate in the literature over the last decade [4]. Recently proposed hypotheses claiming that water is a chain-like liquid [6] or that it is intrinsically heterogeneous by nature [5,7] also challenge the traditional approaches towards the interpretation of water properties and anomalies.

From a theoretical point of view, the investigation of these characteristic structural features of water and the temperature and pressure effects upon them could be achieved by employing multiscale modeling techniques, ranging from classical [8] or quantum ab initio molecular simulation techniques [9] (Molecular Dynamics or Monte Carlo) to coarse-graining ones [10]. In such a way valuable information could be gained and used as a springboard towards a better understanding of water properties.

The dipole moment of water molecules [11-20], as well as the formation of hydrogen bonding (HB) networks among them [19-30], play a crucial role in determining the properties of liquid water. From a theoretical point of view, several methods employing different levels of sophistication have been used so far to provide deeper insights regarding the behavior of the molecular dipole of water [12-18] and polarization effects at several bulk thermodynamic state points.

To provide a more realistic description of electrostatic and polarization effects, as well as dispersion and exchange correlation effects taking place in condensed phases, a detailed description of these interactions in a quantum-mechanical framework 
provides the opportunity to elucidate fundamental questions about their nature. The Car-Parrinello ab initio Molecular Dynamics method [31] offers in principle such a possibility. Using this simulation methodology the dipole moment induced in a water molecule, due to the presence of its neighbor molecules in a bulk environment, can be estimated using more rigorous approaches.

The main aim of the present letter is to reveal the interrelation between the local structural heterogeneities in liquid water and the dipole moment of individual water molecules in different environments. Experimental studies have revealed that the distortions in the local tetrahedral and HB network might have a strong impact on the properties of water even at ambient conditions [5]. In this letter particular attention has been paid to the dependence of the induced dipole on the local tetrahedral order and HB network around each water molecule. Although the average value of the dipole moment of water molecules in the liquid state has been estimated in previous Car-Parrinello studies [17], its dependence on the local environment contributes new important elements to grasp water's peculiar properties in the condensed phase.

\section{Computational Methodology}

A first principles molecular dynamics simulation of liquid water at a fixed ambient density $\left(0.997 \mathrm{~g} / \mathrm{cm}^{3}\right)$ was performed in the framework of the present study, by employing the Car-Parrinello technique. A cubic box with 96 water molecules was used in the simulation and periodic boundary conditions were also employed. The system was pre-equilibrated by performing classical MD for 200 ps, using a flexible water model parametrized from $a b$ initio data [32].

The BLYP density functional [33,34]was used in the calculations, with a plane-wave cutoff set at 80 Ry. A fictitious electronic mass parameter of 400 a.m.u. was also selected in order to study the time evolution of the electronic degrees of freedom. It is known that commonly used density functionals do not describe the long-range dispersion interactions accurately and cannot provide the correct radial dependence of the dispersion interatomic interaction energy. For this reason dispersion-corrected atom-centered pseudopotentials (DCACPs) [35,36] in the Troullier-Martins format [37] for oxygen and hydrogen were also employed to include dispersion interactions. With this method, a dispersion potential is included in the pseudopotential. In this way the electron density coming out of the electronic structure contains the dispersion 
contribution and no empirical parameters need to be added. Previously reported studies have shown that the use of these pseudopotentials successfully accounts for London dispersion forces, reproducing the structural and dynamic properties of water [36], as well as reference interaction energies obtained by high level $a b$ initio calculations.

Following previous studies [36], a 3 ps initial NVT equilibration run at $330 \mathrm{~K}$, followed by a subsequent 15 ps production run at the microcanonical (NVE) ensemble were performed. Our previous studies have also revealed that the effects of finite time and length scale on the obtained structural and dynamic properties of water are not important. Note also that the reference temperature was set during equilibration at 330 $\mathrm{K}$ to avoid falling into the temperature range where simulations with the BLYP functional suffer from non-ergodic behavior on time scales shorter than 20 ps [38].

Maximally localized Wannier functions [39,40] were used to partition the total charge density of the system into individual molecular contributions and to calculate the dipolar moments of each water molecule from the ion and Wannier function center positions [17-18,39-41]. Due to the use of pseudopotentials 8 electrons per water molecule were explicitly considered and hence, due to spin degeneracy, 4 doubly occupied Wannier functions and their relative centers were taken into account $[17,18]$.

\section{Results and Discussion}

A measure of the local orientational arrangement in water can be provided by the tetrahedral order parameter q $[42,43]$. This parameter gives information about the extent to which a molecule and its four nearest neighbors adopt a tetrahedral arrangement and is defined as:

$$
q=1-\left\langle\frac{3}{8} \sum_{j=1}^{3} \sum_{k=j+1}^{4}\left(\cos \phi_{j i k}+\frac{1}{3}\right)^{2}\right\rangle
$$

In this equation $\phi_{j i k}$ corresponds to the angle formed by the vectors $\vec{r}_{i j}$ and $\vec{r}_{i k}$, connecting the oxygen atom of the central molecule i with the oxygen atoms of two of its four nearest neighbours $\mathrm{j}$, $\mathrm{k}$. Using this definition the parameter q gets the value $\mathrm{q}=1$ in a perfect tetrahedral network and the value $\mathrm{q}=0$ in an ideal gas [42]. The calculated normalized probability density distribution function $\mathrm{f}(\mathrm{q})$ is presented in Figure 1. The calculated distribution function exhibits a shoulder around 0.55 and a 
peak at 0.83 . The absence of a unimodal distribution signifies the existence of different "types" of water molecules, exhibiting different local orientational order. To obtain therefore a more quantitative picture of the existing heterogeneities in the local orientational structure in liquid water, the individual water molecules were classified in three types, based on the value of their tetrahedral order parameter at each configuration, as depicted in Figure 1. The first type of water molecules (type A) corresponds to molecules exhibiting a low tetrahedral order with q values less than 0.55. The rest of the molecules, corresponding to the second (strong) mode of the distribution have also been divided into two subcategories. Molecules exhibiting $\mathrm{q}$ values between 0.55 and 0.83 have been categorized as type B and molecules exhibiting a very strong tetrahedral order $(q>0.83)$ have been categorized as type C. Our analysis has revealed that $22.8 \%$ of the molecules in the system are type A, 54.7 $\%$ are type B and $22.5 \%$ are type C ones. From a physical point of view, we could say that roughly half of the molecules in the system are exhibiting either very low or very high tetrahedral order, with equal probabilities, whereas the rest are in an intermediate state.

The intermolecular structure around these different types of water molecules has been investigated in terms of the atom-atom radial distribution functions (rdf). The O-O and $\mathrm{O}-\mathrm{H}$ rdfs around these different types of water molecules, together with the radial dependence of the O-O coordination number are depicted in Figure 2. From this figure it can be observed that type $\mathrm{C}$ and $\mathrm{B}$ molecules form more hydrogen bonds than the type A ones, having also a well-defined first solvation shell. On the other hand, the absence of a clear first minimum in the range of 3-4 $\AA$ in the O-O rdf for type A molecules, signifies the lack of local structural order around these molecules. This behavior indicates the existence of structural and orientational heterogeneities in liquid water.

To have a quantitative picture of the number of hydrogen bonds formed by these different types of molecules, an HB analysis was performed using the following geometric criterion: $\mathrm{R}_{\mathrm{O} \ldots \mathrm{O}} \leq 3.38 \AA, \mathrm{R}_{\mathrm{H} \ldots \mathrm{O}} \leq 2.48 \AA$ and the angle $\phi_{H-O . . \mathrm{O}} \leq 30^{\circ}$ (here ... denotes an intermolecular bond vector, whereas - denotes an intramolecular one). The calculated average numbers of hydrogen bonds formed by type A, B, C water molecules are 2.90, 3.58 and 3.82, respectively. The fractions of water molecules forming 0-5 hydrogen bonds have been calculated for the 3 different types 
and are presented in Figure 3. The fact that the large majority (78.5 \%) of type C molecules form 4 hydrogen bonds supports the fact that the tetrahedral symmetry around water molecules is stabilized due to the formation of these hydrogen bonds. The majority of type A molecules form 2 and 3 hydrogen bonds and therefore the symmetry collapses due to the breaking of this local HB network. The majority of type B molecules form 4 hydrogen bonds, however there is a significant amount forming 3 bonds, leading thus to a decrease of the tetrahedral order parameter. The fraction of molecules forming 5 hydrogen bonds is also higher in the case of type A and $\mathrm{B}$ molecules in comparison with the type $\mathrm{C}$ ones. Since the existence of the tetrahedral network around the water molecules is due to the formation of 4 hydrogen bonds with the neighbor molecules, deviations from this network of 4 hydrogen bonds lead to less tetrahedrally ordered structures. Therefore, it seems very reasonable that in the cases of type A,B molecules the fraction of molecules forming 5 hydrogen bonds is higher than in the case of type $\mathrm{C}$ molecules, which exhibit a strong tetrahedral order around them. All these findings underlie the importance of the HB network on the local structural order in liquid water.

Apart from the static description of the local orientational structural network in liquid water, the dynamics related to the transitions between the A,B and C types of water molecules were studied in terms of the following time decay functions:

$$
C^{X Y}(t)=\frac{\left\langle n^{X}(0) \cdot n^{Y}(t)\right\rangle}{\left\langle n^{X}(0) \cdot n^{Y}(0)\right\rangle}, \quad \mathrm{X}, \mathrm{Y}=\mathrm{A}, \mathrm{B} \text { or } \mathrm{C}
$$

where the parameters $n^{X}(t), n^{Y}(t)$ have been defined as follows:

If at time $\mathrm{t}=0$ a water molecule is in state $\mathrm{X}(\mathrm{A}, \mathrm{B}$ or $\mathrm{C})$, then $n^{X}(0)=1$.

$n^{Y}(t)=1$, if the same molecule is not in state $\mathrm{Y}(\mathrm{A}, \mathrm{B}$ or $\mathrm{C}, \mathrm{Y} \neq \mathrm{X})$.

The first time that this molecule is in state $\mathrm{Y}$, then $n^{Y}(t)=0$ and then for times $t^{\prime}>t$, $n^{Y}\left(t^{\prime}\right)=0$.

The integral of these decay functions up to the point where they reach zero values gives an average estimate of the time required for a transition from one type to another. The calculated decay functions $C^{X Y}(t)$ are presented in Figure 4. The main outcome of this analysis is that type $A$ and $C$ water molecules live shorter than the type B ones and they convert very fast to type B. On the other hand, type B molecules 
convert slower to type $\mathrm{C}$ and $\mathrm{A}$ ones, with the BA transition being faster. The fact that the sum of the transition times $\tau^{A B}+\tau^{B C} \cong \tau^{A C}$ and $\tau^{C B}+\tau^{B A} \cong \tau^{C A}$ indicates that the most probable transition mechanism is:

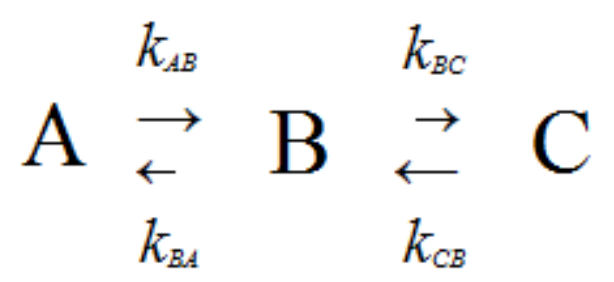

In this mechanism type B acts as an intermediate, longer-lived, structure and the transitions $\mathrm{A} \rightarrow \mathrm{C}$ and $\mathrm{C} \rightarrow \mathrm{A}$ are not direct, but take place through transitions $\mathrm{A} \rightarrow \mathrm{B}$ and $\mathrm{C} \rightarrow \mathrm{B}$ first. Therefore, it seems that fast transitions from low to very high tetrahedrally ordered structures are not promoted in liquid water. It is well known from the literature that the liquid water reorientation mechanism involves largeamplitude angular jumps [44]. The existence of this mechanism was a motivation for us to study for the first time in the literature if these very fast transitions also occur in the local structural order around the water molecules, since these jump reorientational motions (which are also related to the breaking and reforming of the local HB network around the water molecules) could also induce significant changes in the local structural network and allow the direct transfer from "state" A to C and vice versa. However, our findings indicate that no rapid transitions from type A to type $\mathrm{C}$ local environments are observed. The jump mechanism of molecular reorientation takes place when the rotating water molecule breaks a hydrogen bond with an overcoordinated first-shell neighbor to form an H-bond with an undercoordinated second-shell neighbor [44]. However, it seems that although a rapid exchange of $\mathrm{H}$ bonded neighbors takes place through this jump reorientational mechanism, the dynamics of the local environment's tetrahedral symmetry around the rotating molecule are not strongly affected. By considering the time decay of $C^{X Y}(t)$ as an exponential one, the simple assumption that the kinetic constant is the inverse of the transition time can be made and the estimated values for these constants are $\mathrm{k}_{\mathrm{AB}}=9.1$ $\mathrm{ps}^{-1}, \mathrm{k}_{\mathrm{BA}}=2.8 \mathrm{ps}^{-1}, \mathrm{k}_{\mathrm{BC}}=1.7 \mathrm{ps}^{-1}$ and $\mathrm{k}_{\mathrm{CB}}=6.3 \mathrm{ps}^{-1}$. 
The difference in the local structure is reflected in the total dipole moments of distinct water molecules. In Figure 5 the calculated 3-dimensional normalized probability distribution function $\mathrm{P}(\mu, \mathrm{q})$ is depicted together with its corresponding twodimensional contour plot. From this figure it is clear that water molecules having a dipole moment around 3.0 D and a tetrahedral order parameter around 0.8 exhibit the highest probability of appearance. The distribution of the dipole moments for type A,B,C molecules is also presented in the same figure and it is easy to observe that as the tetrahedral order increases the peak of the distribution shifts towards larger dipole moment values. The average values for the dipole moment of type A,B and $C$ water molecules have been estimated to be 2.81, 2.98 and $3.09 \mathrm{D}$, and their standard deviations are $0.27,0.28$ and $0.26 \mathrm{D}$, respectively. Previous studies in the literature have also pointed out the correlation between the water molecular dipole moment and the local environment around them [45, 46]. Interestingly, in a previous study Batista et al. [15] estimated the value of the dipole moment of water molecules in ice Ih to be 3.09 D. In their study they used an induction model including dipole, dipolequadrupole, quadrupole-quadrupole polarizability and first hyperpolarizability as well as fixed octopole and hexadecapole moments to study the electric field in ice. By performing self-consistent calculations they found this average value for the dipole moment of ice Ih water molecules, which is the same with the one calculated for type $\mathrm{C}$ water molecules in the present study. Other studies reported in the literature have also suggested that the dipole moment of water molecules in ice lattices must be larger than 3.0 D $[16,17,47,48]$. A very recent Born-Oppenheimer molecular dynamics simulation study, using the revPBE exchange functional in conjunction with non-local van der Waals correlation [49], also predicted a dipole moment 3.18 D for ice Ih. All these data challenge in this way the earlier reference analysis of Coulson and Eisenberg who predicted a significantly lower value of 2.6 D [14]. According to Batista et al [15], the main reason for this discrepancy was the use of less accurate values for the molecular multipoles in these earlier calculations.

Taking into account that the fourth nearest neighbors of a water molecule in ice Ih are geometrically arranged in such a way giving an almost perfect tetrahedral symmetry, our result reveals that this local symmetry is the main factor controlling the value of the dipole moment. So even at the liquid state, the small fractions of molecules which retain this almost perfect tetrahedral symmetry maintain the same dipole moment as ice Ih molecules. 
In order to check about the existence of different kinds of local structures around the water molecules, the trigonal and trigonal bipyramidal order parameters [50], $\mathrm{q}_{3}$ and $\mathrm{q}_{5}$ respectively, were also studied as a function of the number of hydrogen bonds. The $\mathrm{q}_{3}$ and $\mathrm{q}_{5}$ order parameters have been recently introduced in the study of Henchman and Cockram [50] and detailed information about these order parameters can be found there. The $\mathrm{q}_{3}$ order parameter is defined, taking into account the three closest neighbors of a molecule i, as:

$$
q_{3}=1-\left\langle\frac{4}{7} \sum_{j=1}^{2} \sum_{k=j+1}^{3}\left(\cos \phi_{j i k}+\frac{1}{2}\right)^{2}\right\rangle
$$

and the $\mathrm{q}_{5}$ order parameter has been defined, taking into account the five closest neighbors of a molecule i, as:

$$
q_{5}=1-\left\langle\frac{6}{35} \sum_{j=1}^{2} \sum_{k=j+1}^{3}\left(\cos \phi_{j i k}+\frac{1}{2}\right)^{2}\right\rangle-\left\langle\frac{3}{10} \sum_{l=1}^{2} \sum_{j=1}^{3}\left(\cos \phi_{l i j}\right)^{2}\right\rangle-\left\langle\frac{3}{40}\left(\cos \phi_{m i l}+1\right)^{2}\right\rangle
$$

where $\mathrm{j}, \mathrm{k}$ are indices for the 3 equatorial neighbors of the central $\mathrm{i}$ molecule,defining thus 3 equatorial-i-equatorial angle cobinations (first term of equation 4), and l, m are indices for the remaining two axial neighbors, defining thus 6 axial-i-equatorial angle combinations (second term of equation 4) and one axial-i-axial angle (third term). According to the definition of Henchman and Cockram [50], the three equatorial oxygens in the case of $\mathrm{q}_{5}$ are those whose angles between their $\mathrm{OO}$ vector and the water molecule's $\mathrm{HOH}$ plane are closest to zero. The remaining two are axial.

As in the case of the tetrahedral order parameter q, these order parameters have been normalized to one for perfect order and zero for a random distribution. The dependence of the $\mathrm{q}, \mathrm{q}_{3}$ and $\mathrm{q}_{5}$ order parameters on the number of hydrogen bonds formed by a water molecule is presented in Figure 6a. From this figure it can be seen that the $\mathrm{q}_{5}$ values are much lower than the $\mathrm{q}_{3}$ and $\mathrm{q}$ ones, signifying that the trigonal bipyramidal structure is not being favored in liquid water. Even in the case of water molecules forming 5 hydrogen bonds, where the $q_{5}$ attains its maximum value, the 
average value of $\mathrm{q}_{5}$ is 0.53 , quite far from the value of 1 where the perfect trigonal bypyramidal order is observed. Interestingly, it was also observed from our calculations that the distribution of $\mathrm{q}_{5}$ for molecules forming 5 hydrogen bonds is bimodal, with one peak observed at 0.4 and the second at 0.8 . All the rest of the distributions, for molecules forming less than 5 hydrogen bonds, are monomodal with peaks at low $\mathrm{q}_{5}$ values. Even in the case of molecules forming 5 hydrogen bonds, only $12 \%$ of these molecules exhibit $\mathrm{q}_{5}$ higher than 0.8 . This finding signifies that even in the cases where the water molecules form 5 hydrogen bonds the trigonal bypyramidal structure is not the preferred structure and it is in general quite rarely observed in liquid water.

On the other hand, the $\mathrm{q}_{3}$ values are the highest ones and interestingly they are maximized in the case of water molecules forming 4 hydrogen bonds, where they have a very similar value with the tetrahedral order parameter q. This finding is not peculiar, since in a tetrahedron the three of the four neighbors of a water molecule form a triangle. The fact however, that in general the $q_{3}$ values are higher than the $q$ ones was a motivation to study if the trigonal structure is the predominant one for type A and $B$ molecules. The average $\mathrm{q}_{3}$ values for type A,B and C water molecules have been found to be $0.59,0.77$ and 0.86 , respectively. The fact that the average $\mathrm{q}_{3}$ value for type A molecules is quite low and far from one, indicates the absence of a strong orientational ordering around type A molecules. On the other hand, it seems that for type B molecules the trigonal local ordering is more important, even though the fraction of molecules forming 3 hydrogen bonds is still the dominant one (see Figure 3). Therefore it seems that the trigonal order in type B molecules is induced mainly due to the breaking of the fourth hydrogen bond, leading thus to molecules forming 3 hydrogen bonds and due to the existence of distorted tetrahedral structures of molecules which still form 4 hydrogen bonds. The fact that $\mathrm{q}_{3}$ is even larger for type C molecules can be explained, as mentioned above, due to the fact that the trigonal local order is a subset of the tetrahedral one. Therefore, type $\mathrm{C}$ molecules can be characterized as tetrahedral one and not trigonal.

As mentioned above, we found that HB interactions are responsible for the local symmetry around the water molecules and that the very large majority of type $C$ molecules form 4 hydrogen bonds. In order to further verify the strong coupling between hydrogen bond formation, local tetrahedral symmetry and the molecular dipole moment of water, the dependence of the dipole moment on the number of 
hydrogen bonds formed by each water molecule was investigated and is presented in Figure 6b. From this figure it can be seen that the dipole moment of a water molecule significantly increases with the increase of the number of hydrogen bonds which forms with its neighbors. Therefore, for hydrogen bonded free molecules the calculated average molecular dipole moment is $2.26 \mathrm{D}$ and for molecules forming 4 hydrogen bonds the corresponding values is $3.07 \mathrm{D}$. The latter one is essentially the same with the value obtained for type $C$ water molecules and ice Ih ones.

The dipole moments of molecules with very high q values (type C) which form 4 hydrogen bonds and the dipole moments of molecules with very high $\mathrm{q}_{5}$ values (higher than 0.8) which form 5 hydrogen bonds were also calculated. Interestingly these dipole moments are almost similar (3.14 and 3.15 D, respectively). The average dipole moment of water molecules forming 5 hydrogen bonds and having lower $\mathrm{q}_{5}$ values are also not affected by the lack of the trigonal bipyramidal symmetery and are similar (3.12 D). Therefore it seems that even in a more condensed and symmetrical environment like the one where the central water molecule is surrounded by 5 neighbors and has trigonal bipyramidal symmetry, the dipole moment of the central water molecule does not increase (although it would be expected to be higher) in comparison with molecules having a tetrahedral packing around them. Actually such a fact indicates the strong coupling between HB interactions, tetrahedral order and the molecular dipole moment of water.

\section{Conclusions}

The local structural heterogeneities in ambient water were investigated and related to the existing dipolar fluctuations in the liquid by means of Car-Parinello quantum molecular dynamics simulations. Special attention was given to the local tetrahedral order, molecular dipole fluctuations and their interrelation with hydrogen bonding interactions in the liquid. Our analysis revealed that almost half of the molecules in the system are exhibiting either very low or very high tetrahedral order, with equal probabilities, whereas the rest are in an intermediate state. This intermediate state exhibits the largest lifetime and transitions from low to very high tetrahedrally ordered structures take place only through transitions to this state. The degree of hydrogen bonding also increases with the increase of the tetrahedrality, supporting the 
fact that the tetrahedral symmetry around water molecules is stabilized due to the formation of hydrogen bonds.

The dependence of the dipole moment of water molecules on their local environment has also been investigated, revealing the increase of the dipolar moment for molecules exhibiting higher tetrahedral order parameters. Water molecules having a dipole moment around 3.0 D and a tetrahedral order parameter around 0.8 exhibit the highest probability of appearance in the liquid. Interestingly, the calculated average dipole moment (3.09 D) of water molecules exhibiting a strong tetrahedral order around them comes in excellent agreement with previous estimations of the dipole moment of ice Ih molecules. This result comes also in agreement with the value obtained for molecules forming 4 hydrogen bonds, confirming the strong coupling between HB interactions, local tetrahedral order and the molecular dipole moment of water.

\section{Acknowledgements}

The authors thankfully acknowledge the computer resources, technical expertise, and assistance provided by the Barcelona Supercomputing Center, Centro Nacional de Supercomputación, for the projects QCM-2009-1-0014, QCM-2008-3-0012, and QCM-2008-2-0010. The research institution INSTM is also acknowledged by M.M., who is also thankful for the resources given by the Cybersar Project managed by the “Consorzio COSMOLAB”. E.G. acknowledges financial support from the Ministerio de Economía y Cometitividad (MINECO) of Spain (Grant FIS2012-39443-C02-01). I.S. acknowledges the HPC-EUROPA 2 project (project 228398) for the support of the European Commision-Capacities Area-Research Infrastructures, which allowed him to visit the group of M.M. in the University of Sassari and carry out part of the analysis presented in this work.

\section{References}

[1] D. Eisenberg, W. Kauzmann, The structure and properties of water, Oxford University Press, New York, 1969

[2] F. Franks, Water: A Comprehensive Treatise Vol.1, Plenum Press, New York, 1975 
[3] P. Ball, Nature, 452 (2008) 291

[4] P. Wernet, D. Nordlund, U. Bergmann, M. Cavalleri, M. Odelius, H. Ogasawara, L.A. Naslund, T. K. Hirsch, L. Ojamae, P. Glatzel, L.G.M. Pettersson, A. Nilsson, Science 304 (2004) 995

[5] C. Huang, K.T. Wikfeldt, T. Tokushima, D. Nordlund, Y. Harada, U. Bergmann, M. Niebuhr, T.M. Weiss, Y. Horikawa, M. Leetmaa, M.P. Ljungberg, O. Takahashi, A. Lenz, L. Ojamae, A.P. Lyubartsev, S. Shin, L.G.M. Pettersson and A. Nilsson, Proc. Natl. Acad. Sci. (USA) 106 (2009) 15214

[6] A.K. Soper, Pure Appl. Chem. 82 (2010) 1855

[7] A. Nilsson, L. G. M. Pettersson, Chem. Phys. 389 (2011) 1

[8] B. A. Guillot, J. Mol. Liq. 101 (2002) 219

[9] F. Paesani, G. A. Voth, J. Phys. Chem. B 113 (2009) 5702

[10] K. R. Hadley, C. McCabe, Mol. Simul. 38 (2012) 671

[11] S. A. Clough, Y. Beers, G.P. Klein, L.S. Rothman, J. Chem. Phys. 59 (1973) 2254

[12] S. S. Xantheas, T.H. Dunning, Jr., J. Chem. Phys. 99 (1993) 8774

[13] J.K. Gregory, D.C. Clary, K. Liu, M.G. Brown, R.J. Saykally, Science 275 (1997) 814

[14] C.A. Coulson, D. Eisenberg, Proc. R. Soc. London A 291 (1966) 445

[15] E. R. Batista, S. S. Xantheas, H. Jónsson, J. Chem. Phys. 109 (1998) 4546

[16] D.J. Adams, Nature 293 (1981) 447

[17] P.L. Silvestrelli, M. Parrinello, Phys. Rev. Lett. 82 (1999) 3308

[18] D. Pan, L. Spanu, B. Harrison, D.A. Sverjensky, G. Galli Proc. Natl. Acad. Sci. (USA) 110 (2013) 6646

[19] Y.S. Badyai, M.-L. Saboungi, D.L. Price, S.D. Shastri, D.R. Haeffner, A.K. Soper, J. Chem. Phys. 112 (2000) 9206 
[20] D. Akase, M. Aida, J. Phys. Chem. A 118 (2014) 7911

[21] E. Guardia, I. Skarmoutsos, M. Masia, J. Phys. Chem. B 119 (2015) 8926

[22] A.K. Soper, J. Phys. Chem. B 115 (2011) 14014

[23] R.H. Henchman, S.J. Irudayam, J. Phys. Chem. B 114 (2015) 16792

[24] Y. Marechal, The hydrogen bond and the water molecule, Elsevier, Oxford, UK, 2007

[25] D. Swiatla-Wojcik, J. Szala-Bilnik, J. Chem. Phys. 134 (2011) 054121

[26] I. Skarmoutsos, E. Guardia, J. Chem. Phys. 132 (2010) 074502

[27] L. Liang, P. Rulis, L. Ouyang, W.Y. Ching, Phys. Rev. B 83 (2011) 024201

[28] T. Head-Gordon, M.E. Johnson, Proc. Natl. Acad. Sci. (USA) 103 (2006) 16615

[29] F. Stillinger, Science 209 (1980) 451

[30] J. D. Smith, C.D. Cappa, K.R. Wilson, R.C. Cohen. P.L. Geissler, R.J. Saykally Proc. Natl. Acad. Sci. (USA) 102 (2005) 14171

[31] R. Car, M. Parrinelllo, Phys. Rev. Lett. 55 (1985) 2471

[32] O. Akin-Ojo, F. Wang, J. Chem. Phys. 129 (2008) 064108

[33] A. Becke, Phys. Rev. A 38 (1988) 3098

[34] C. Lee, W. Yang, R. Parr, Phys. Rev. B 37 (1988) 785

[35] O.A von Lilienfeld, I. Tavernelli, U. Röthlisberger, D. Sebastiani, Phys. Rev. Lett. 93 (2004) 153004

[36] I.-C. Lin, A.P. Seitsonen, M.D. Coutinho-Neto, I. Tavernelli, U. Röthlisberger, J. Phys. Chem. B 113 (2009) 1127

[37] N. Troullier, J.L. Martins, Phys. Rev. B 43 (1991) 1993

[38] I.-F. W. Kuo, C.J. Mundy, M.J. McGrath, J.I. Siepmann, J. VandeVondele, M. Sprik, J. Hutter, B. Chen, M.L. Klein, F. Mohamed, J. Phys. Chem. B 108 (2004) 12990 
[39] N. Marzari, D. Vanderbilt, Phys. Rev. B 56 (1997) 12847

[40] P.L. Silvestrelli, N. Marzari, D. Vandebilt, M. Parrinello, Solid State Commun. 107 (1998) 7

[41] E. Guardia, I. Skarmoutsos, M. Masia, J. Chem. Theory Comput. 5 (2009) 1449

[42] J. R. Errington, P.G. Debenedetti, Nature, 409 (2001) 318

[43] C. Zhang, L. Spanu, G. Galli, J. Phys. Chem. B 115 (2011) 14190

[44] D. Laage, J. T. Hynes, Science 311 (2006) 832

[45] M. J. McGrath, J. I. Siepmann, I.-F. W. Kuo, C. J. Mundy, J. VandeVondele, J. Hutter, F. Mohamed, M. Krack, J. Phys. Chem. A 110 (2006) 640

[46] M. J. McGrath, J. I. Siepmann, I.-F. W. Kuo, C. J. Mundy, Mol. Phys. 105 (2007) 1411

[47] M.I. Heggie, C.D. Latham, S.C.P. Maynard, R. Jones, Chem. Phys. Lett. 249 (1996) 485

[48] P.A.F.P. Moreira, M. de Koning, Phys. Chem. Chem. Phys. (2015) DOI: 10.1039/C5CP03346B

[49] N.J. English, Energies 8 (2015) 9383

[50] R. H. Henchman, S. J. Cockram, Faraday Discuss. 167 (2013) 529 


\section{FIGURES}

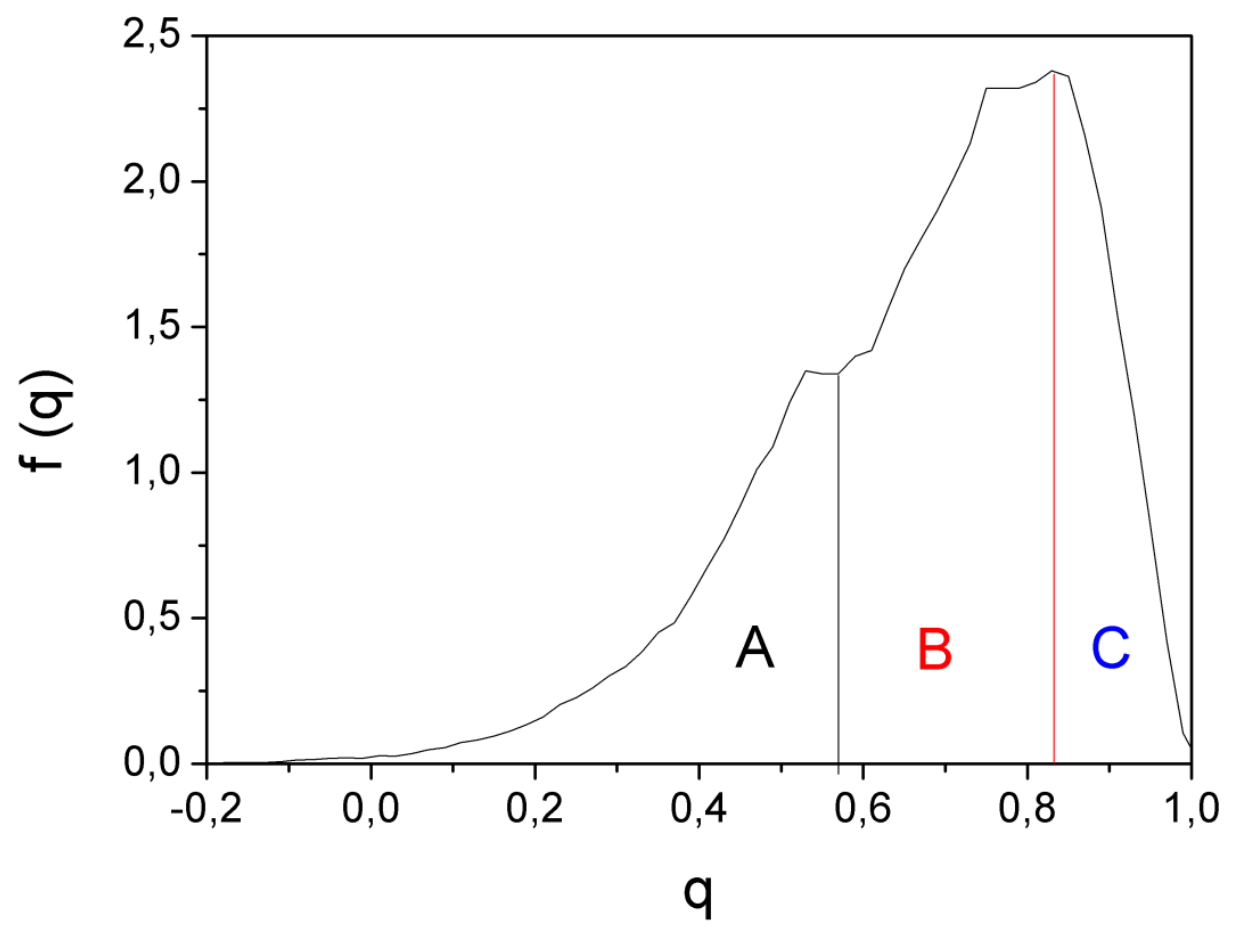

Figure 1 

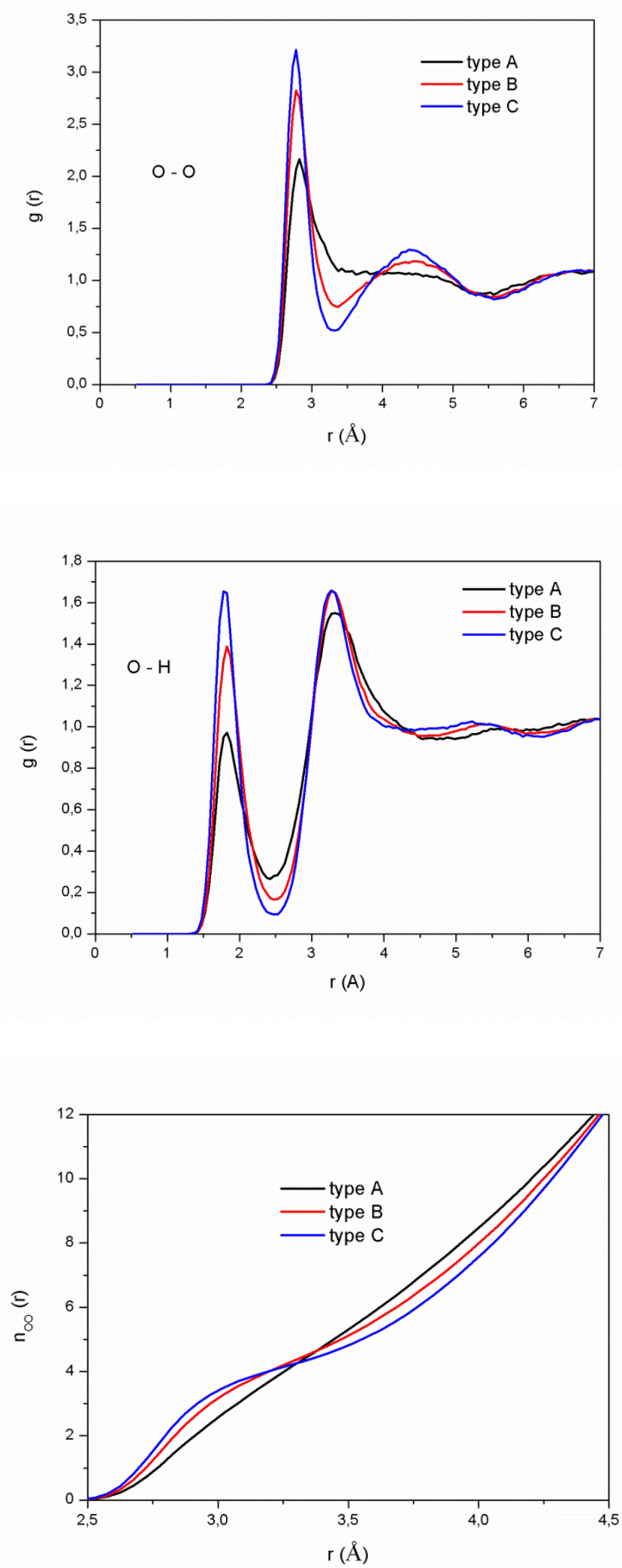

Figure 2 


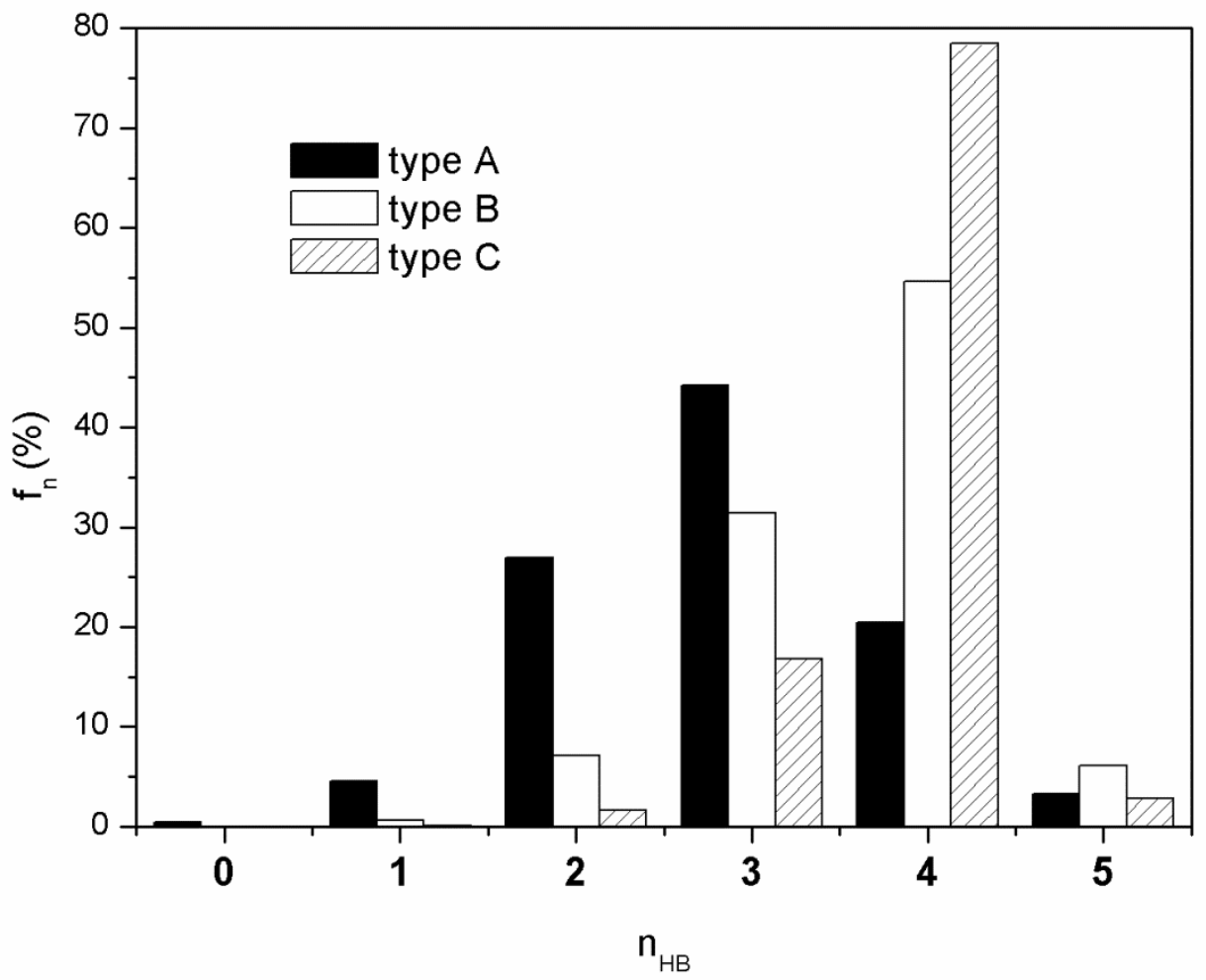

Figure 3 


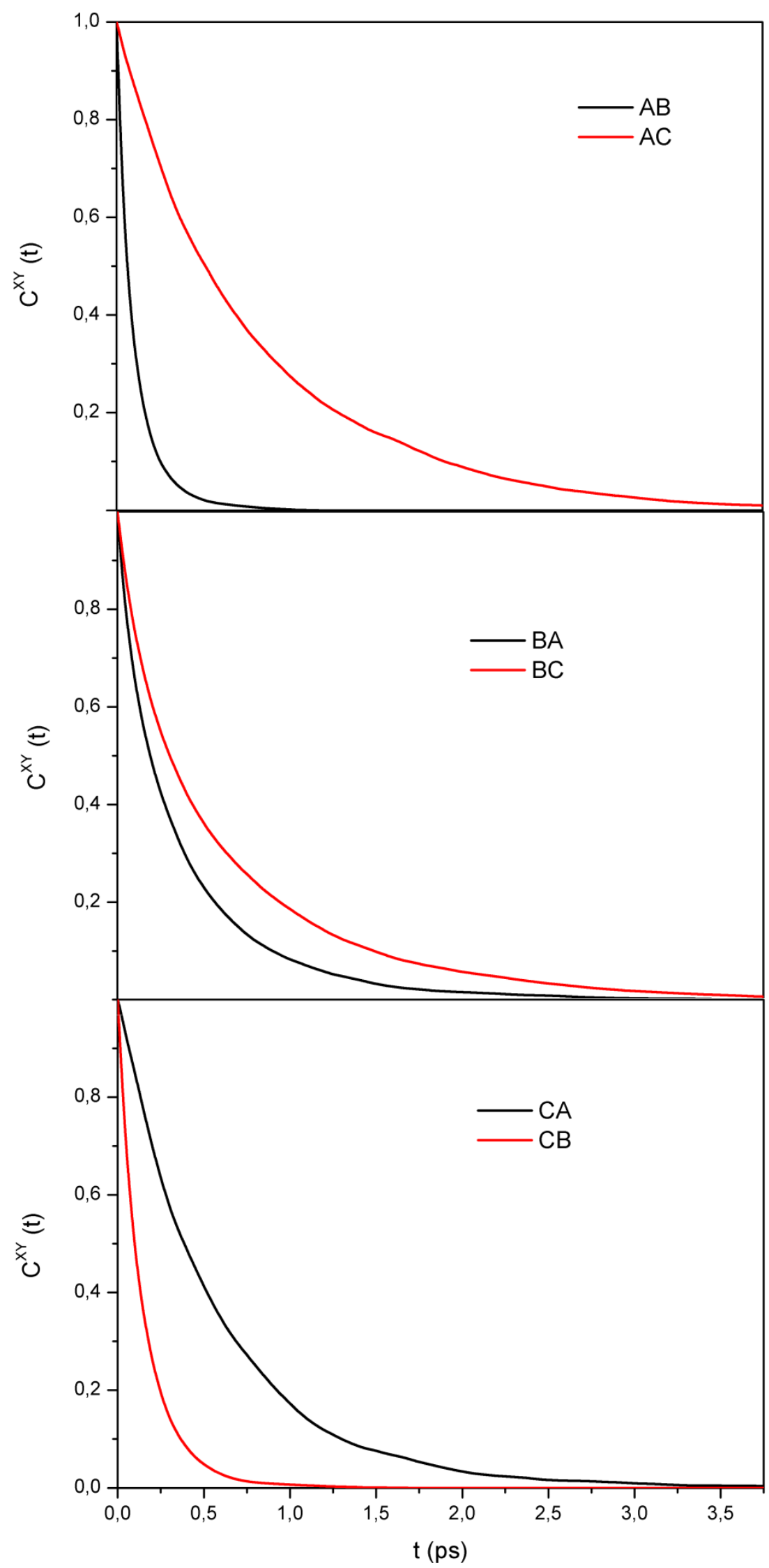

Figure 4 

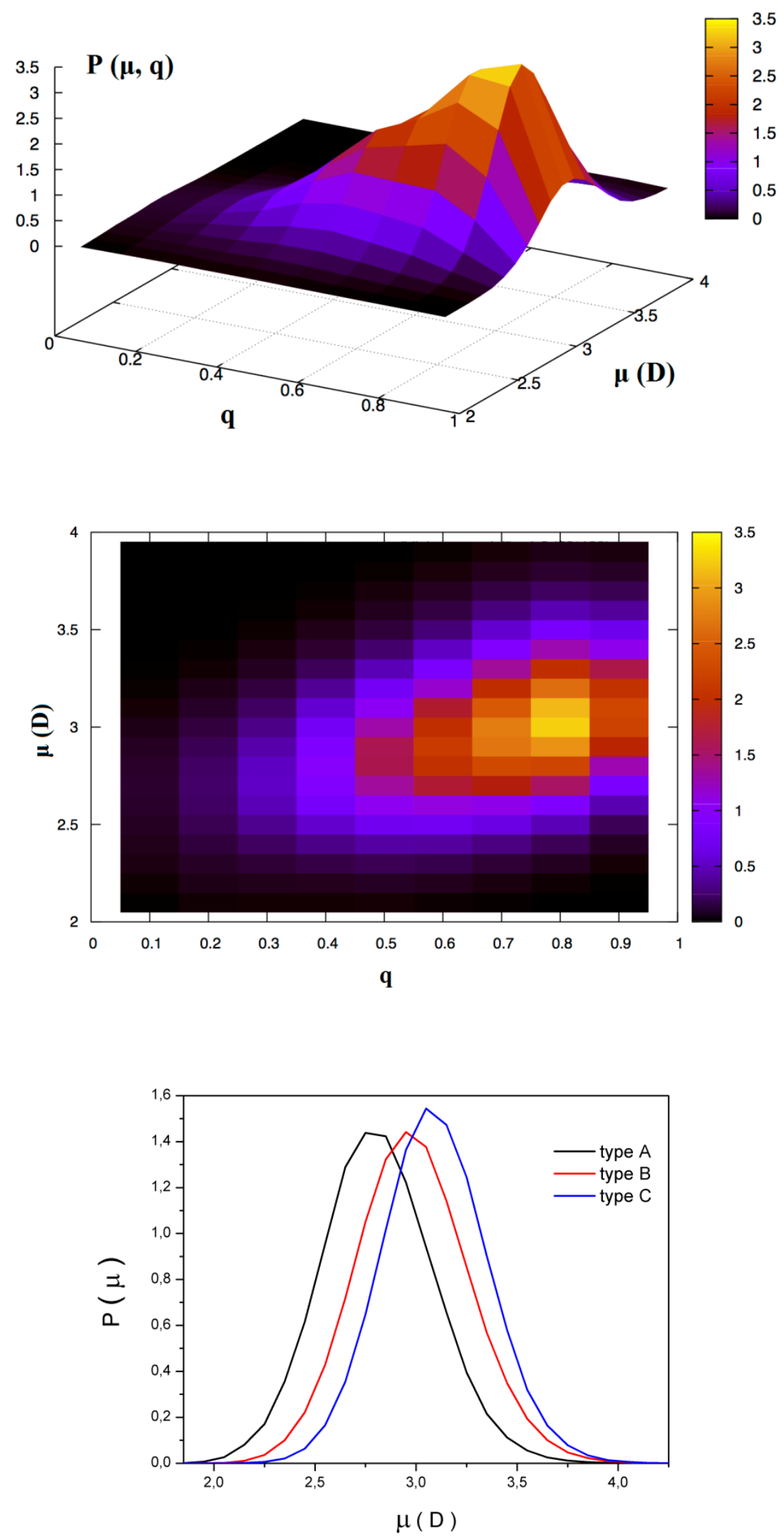

Figure 5 

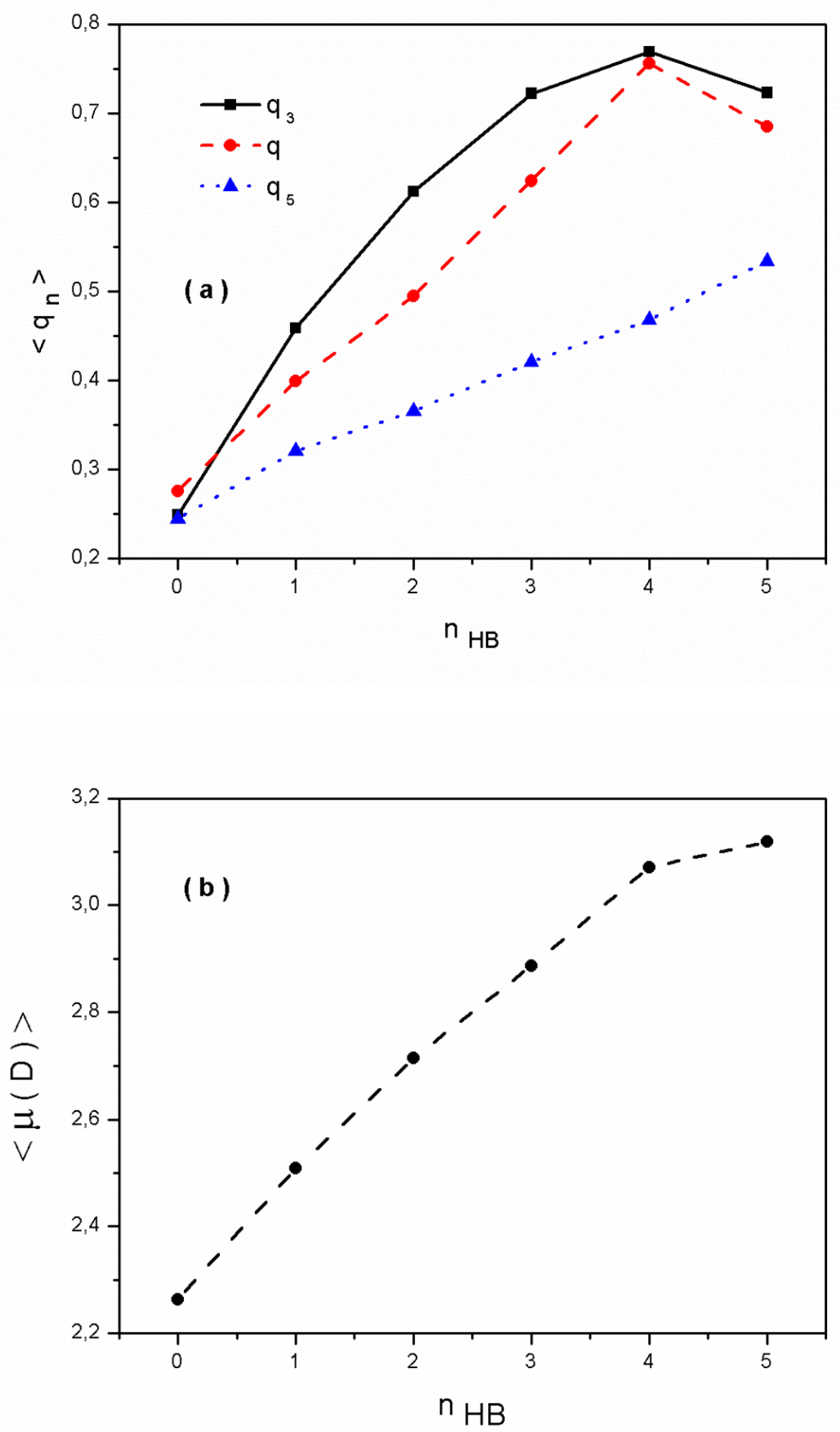

Figure 6 


\section{Figure Captions}

Figure 1: Classification of water molecules based on the distribution of the tetrahedral order parameter.

Figure 2: $\mathrm{O}-\mathrm{O}$ and $\mathrm{O}-\mathrm{H}$ radial distribution functions and $\mathrm{O}-\mathrm{O}$ coordination number of the three different types A, B, C of water molecules.

Figure 3: Hydrogen bond statistics for the three different types A, B, C of water molecules.

Figure 4: Time decay functions related to the transitions between the three types A, $\mathrm{B}, \mathrm{C}$ of water molecules.

Figure 5: Calculated 3-dimensional normalized probability distribution function $\mathrm{P}(\mu, \mathrm{q})$ and 2-dimensional normalized probability distribution $\mathrm{P}(\mu)$ for the three different types A, B, C of water molecules.

Figure 6: (a) Dependence of the $\mathrm{q}, \mathrm{q}_{3}$ and $\mathrm{q}_{5}$ order parameters on the number of hydrogen bonds formed by each water molecule (b) Dependence of the molecular dipole moment on the number of hydrogen bonds formed by each water molecule. 